M. SAKAI

KODAI MATH. J.

01 (1987), 39-41

\title{
A UNIQUENESS THEOREM FOR MINIMAL SURFACES IN $S^{3}$
}

\author{
BY MAKOTO SAKAKI
}

\section{$\S 1$. Introduction.}

$R^{3}$ and $S^{3}$ have some similar properties. First both of them have congruent translations such as the parallel translations or the rotations, and secondly they have the concept of the convex hull. ([1]).

W. H. Meeks III states some uniqueness theorems for minimal surfaces in $R^{3}$ and one of the theorems is the following.

THEOREM 1 (Meeks III [3]). Suppose $\gamma$ is a $C^{2}$-Jordan curve on a plane. Then there exists a positive number $\varepsilon$ so that any Jordan curve in $R^{3}$ which is $\varepsilon$ close to $\gamma$ in the $C^{2}$-norm is the boundary curve of a unique compact minimal surface. Furthermore, this minmal surface is a graph over the plane.

We will show anologous theorem paying attention to the next paragraph for minimal surfaces in $S^{3}$.

THEOREM 2. Suppose that $\gamma$ is a $C^{2}$-Jordan curve on a geodesic 2-sphere in $S^{3}$ and belongs to some open hemisphere of $S^{3}$. Then there exists a positive number $\varepsilon$ such that any Jordan curve in $S^{3}$ which is $\varepsilon$ close to $\gamma$ in the $C^{2}$-norm is the boundary curve of a unique compact minimal surface in the open hemisphere. And also this minimal surface can be represented as a graph over the geodesic 2-sphere.

\section{§. Preparation.} in $[2]$.

We introduce for our arguement the following model for $S^{3}$ which is found

We identify $R^{3}$ which $S^{3}$ by the stereographic projection from the point $(0,0,0,-1)$. The origin $O=(0,0,0)$ corresponds to the south pole of the projection. The geodesics of $S^{3}$ correspond to the all straight lines through $O$, or all great circles of the unit sphere $S$ centered at $O$, or all plane circles meeting $S$ in antipodal points. The geodesic 2 -spheres of $S^{3}$ correspond to all planes through $O$, or the sphere $S$, or all Euclidean spheres which meet $S$ in a great

Received March 7, 1986 
circle of $S$. All plane circles correspond to the small circles in $S^{3}$. So consider all plane circles which intersect orthogonally with $x y$-plane $P$ and $S$. Then we find some translation along this flow of circles corresponds to a rotation which leaves $C=$ the intersection of $P$ and $S$ fixed.

\section{§3. Proof of the theorem 2.}

Proof. We use the same method as [3]. By the assumption of the theorem we shall assume that $\gamma$ is on $P$ and belongs to the interior of $C$, which is the argument in the model of $S^{3}$. Let $F=\left\{\gamma_{t} \mid 0 \leqq t \leqq 1\right\}$ be a $C^{2}$-foliation by Jordan curves of the annular region in $P$ bounded by $\gamma_{0}=\gamma$ and $\gamma_{1}=C$.

Consider a point which goes from each point on $\gamma_{t}$ along the geodesic $m$ on $P$ in the direction of the inner normal by $\beta$. Here $\beta$ is a small positive number so that for each $t$ we obtain another Jordan curve $\alpha_{t}$ as a set of the points above. Suppose that on a geodesic 2-sphere two geodesics intersect orthogonally at some point $Q$, and let $\delta(\beta)$ be the distance between the two points each of which is on each of the two geodesics and is far from $Q$ by $\beta$. Consider a point which goes above (below) by $\beta$ from each point on $\alpha_{t}$ along the geodesic $n$ through the point that is orthogonal to $P$, and around this point construct a geodesic circle of radius $\delta(\beta)$ on the geodesic 2 -sphere made by $m$ and $n$. By these constructions we obtain the torus $T_{t}^{+}\left(T_{t}^{-}\right)$which is around $\alpha_{t}$ and contains $\gamma_{t}$ for each $t$. Here we put a new restriction on $\beta$. The number $\beta$ should be small enough for $T_{t}^{+}$(or $T_{t}^{-}$) to have positive mean curvature with respect to the inner normal.

Take two points $X^{+}, X^{-}$on $z$-axis which are far from $O$ by $\delta(\beta)-\beta$ and construct two geodesic 2-spheres $P^{+}, P^{-}$including $C$ and $X^{+}$or $X^{-}$. Let $S_{t}$ be a piecewise smooth sphere constructed by $T_{t}^{+}, T_{t}^{-}, P^{+}$and $P^{-}$, which contains $\gamma_{t}$. Then there are subsets $A_{t}^{+}, \boldsymbol{A}_{t}^{-}, D_{t}^{+}$and $D_{t}^{-}$of $T_{\bar{t}}^{-}, T_{t}^{+}, P^{+}$and $P^{-}$such that $S_{t}$ is the union of $A_{t}^{+}, A_{\bar{t}}^{-}, D_{t}^{+}$and $D_{t}^{-}$.

Let $G=\left\{f: S^{3} \rightarrow S^{3} \mid f\right.$ is a $C^{2}$-diffeomorphism $\}$ and

$$
N=\{f \mid f \text { is contained in } G \text { and satisfies (1) (2) (3) }\} .
$$

(1) $\|f(x)-x\|<\xi$ for all points $x$ in $S^{3}$.

(2) $\left(D f_{x}(v) /\left\|D f_{x}(v)\right\|, v\right)>\cos \eta$ for all $x$ in $S^{3}$ and $v$ in $T_{x} S^{3}$ with $\|v\|=1$.

(3) $f$ (int $A_{t}^{+}$) and $f\left(\right.$ int $A_{\bar{t}}^{-}$) have positive mean curvature for all $t$.

Here we take $\xi>0$ such that $\xi$-neighborhood of $P^{+}$(or $P^{-}$) and the convex hull of $\xi$-neighborhood of $\gamma$ are disjoint and set

$\eta=\min \{$ the angle between $v$ and the tangent vector at $x$ of the small circle through $x$ which is orthogonal to $P$ and $S\}$.

Here we take minimum among all $x$ in $A_{0}^{+}$and $v$ in $T_{x} S_{0}$. Construct the co- 
ordinate system in $S^{3}$ by the flow of small circles orthogonal to $P$ and $S$, and by the coordinate system on the geodesic 2 -spheres orthogonal to the flow. The inner product in (2) is the inner product of $R^{3}$ in such coordinate. $N$ is an open neighborhood of identity map in $G$.

Let $M$ be a minimal surface in $S$ with boundary $f(\gamma)$ where $f$ is contained in $N$. We will first show that $M$ is contained in the ball $B$ bounded by $f\left(S_{0}\right)$. If $M$ is not contained in $B$, then $\sigma=\max \{t \mid 0 \leqq t \leqq 1$, the intersection of $M$ and $f\left(S_{t}\right)$ is not empty $\}$ is greater than 0 . By condition (1), the convex hull of $f(\gamma)$ is contained between $f\left(P^{+}\right)$and $f\left(P^{-}\right)$. This shows that the intersection of $M$ and $f\left(S_{\sigma}\right)$ is contained in the union of $f\left(A_{\sigma}^{+}\right)$and $f\left(A_{\sigma}^{-}\right)$. And also by condition (1), $M$ is contained in the interior of the ball bounded by $f\left(S_{1}\right)$. Thus $\sigma<1$. Condition (2) implies that the interior angles along the surfaces $f\left(A_{\sigma}^{+}\right)$and $f\left(A_{\bar{\sigma}}\right)$ are less than $\pi$. Hence the intersection of $M$ and $f\left(S_{\sigma}\right)$ is contained in the union of $f$ (int $A_{\sigma}^{+}$) and $f$ (int $A_{\sigma}^{-}$). We find this fact is a contradiction by condition (3) and by maximum principle. So we obtain $\sigma=0$ and $M$ is contained in $B$, and also int $M$ is contained in int $B$.

We regard the flow of small circles orthogonal to $P$ and $S$ as vertical lines. Condition (2) implies that $f$ (union of $A_{0}^{+}$and $D_{0}^{+}$) and $f$ (union of $A_{0}^{-}$and $D_{0}^{-}$) are graphs over $P$ in the above sence. This fact and the fact that int $M$ is contained in int $B$ show that the nontrivial rotation of $M$ along the flow of small circles orthogonal to $P$ and $S$ is disjoint from $f(\gamma)$, which is essential.

If $M$ is not a graph over $P$, then some nontrivial rotation of int $M$ intersects int $M$ and locally above it. By using maximum principle we can lead a contradiction. Thus $M$ is a graph over $P$. The same method leads a contradiction if there are two distinct minimal surfaces in $S$ with boundary $f(\gamma)$. Thus $f(\gamma)$ is the boundary of a unique compact minimal surface in $S$. q. e.d.

\section{REFERENCES}

[1] H. B. Lawson, JR., The global behavior of minimal surfaces in $S^{n}$, Ann. Math., 92 (1970), 224-237.

[2] H. B. Lawson, JR., Complete minimal surfaces in $S^{3}$, Ann. Math., 92 (1970), 335-374.

[3] W. H. MeEks III, Uniqueness theorems for minimal surfaces, Illinois J. Math., 25 (1981), 318-336. 